\title{
Study of the population inversion mechanisms and superradiance on ion transitions of molecular nitrogen in the filament
}

\author{
N.G. Ivanov ${ }^{1}$, V.F. Losev ${ }^{1,2}$, V.E. Prokop'ev ${ }^{1,3}$ \\ ${ }^{1}$ Institute of High Current Electronics SB RAS, Akademichesky Avenue 2/3, 634055, Tomsk, \\ Russia, losev@ogl.hcei.tsc.ru \\ ${ }^{2}$ National Research Tomsk Polytechnic University, Lenin Avenue 30, 634050, Tomsk, Russia \\ ${ }^{3}$ National Research Tomsk State University, 36, Lenina Ave., 634050, Tomsk, Russia
}

\begin{abstract}
The experimental results of the inversion population mechanisms study in the resonant electronic transition $\mathrm{B}^{3} \Pi_{\mathrm{g}}-\mathrm{A}^{3} \Sigma_{\mathrm{u}}^{+}$ of nitrogen ions by optically pumped of air and pure nitrogen by femtosecond laser pulse at a wavelength of $950 \mathrm{~nm}$ are presented. It is shown that the inversion results from selective settling of $\mathrm{N}_{2}^{+}\left(\mathrm{B}^{2} \Sigma_{\mathrm{u}}^{+}, \mathrm{v}^{\prime}=0\right)$ excited state by multiphoton excitation of the autoionization states of the nitrogen molecule with energy of $18.7 \mathrm{eV}$. Seed photon for superradiance at transitions of molecular nitrogen ions are photons the axial supercontinuum occurring in the filament on the respective wavelengths. The mode of the superradiance at a wavelength $\lambda=358.4 \mathrm{~nm}$ referred to the transition of the $\mathrm{CN}$ molecules was realized.
\end{abstract}

Keywords: inversion population, mechanisms, filament, femtosecond laser pulse, multiphoton ionization, molecular, nitrogen

\section{INTRODUCTION}

For the first time lasing in nitrogen it was obtained in 1963 in a pulsed discharge plasma of low pressure in the transitions of first $\left(B^{3} \Pi_{g}-A^{3} \Sigma_{u}^{+}\right.$, the wavelength $\left.\lambda=1046.9\right)$ and second $\left(C^{3} \Pi_{u}-B^{3} \Pi_{g}, \lambda=337.1 \mathrm{~nm}\right)$ positive systems of molecular nitrogen [1]. Later the generation on these lines was obtained by the ignition of a discharge in air at atmospheric pressure [2, 3]. In 1974 the generation in most of vibrational-rotational transitions of the second positive system of nitrogen was implemented when a high pressure $\mathrm{N}_{2}-\mathrm{Ar}$ mixture was pumped by an electron beam [4]. Generation at 391.4 and $427.8 \mathrm{~nm}$ wavelengths of the first negative system of molecular nitrogen ion $\left(\mathrm{B}^{2} \Sigma_{\mathrm{u}}^{+}-\mathrm{X}^{2} \Sigma_{\mathrm{g}}{ }^{+}\right)$ was first obtained by electron-beam pumping in 1974 [5] and pumped by a pulsed discharge in 1975 [6].

The distinctive feature of the above generating lines for all kinds of excitation is the large gains. Therefore they can generate in a superradiance mode when the active medium length of the order of a few centimeters without cavity.

It is for this reason the first superradiance on the second positive system of nitrogen molecules in the filament by femtosecond laser (FL) beam pumping with a wavelength of $800 \mathrm{~nm}$ of air was obtained in 2003 [7]. Then it was confirmed by FL pumping of air [8] and a nitrogen and argon mixture [9].

In 2011 lasing on $\mathrm{B}^{2} \Sigma_{\mathrm{u}}^{+}-\mathrm{X}^{2} \Sigma_{\mathrm{g}}^{+}$transitions in molecular nitrogen ion emitting at wavelengths of 391.4 and $427.8 \mathrm{~nm}$ in the forward direction was obtained by pumping a short and intense infrared laser pulse at a wavelength in the middle infrared region $(\lambda=1.2 \div 2.9 \mu \mathrm{m})$ [10]. For lasing at the aforementioned wavelengths the wavelength of the third or fifth harmonics of a pump laser should be coincided with them. It was later shown that the generation at these transitions occur and with femtosecond laser pump at a $800 \mathrm{~nm}$ wavelength in the presence of a second laser operating at a wavelength near $400 \mathrm{~nm}[11,12]$. In the latter case the second laser is an analog of the third or fifth harmonic of pump laser operating in the mid-IR.

In 2013 in our paper $[13,14]$ it was shown that super-radiance at the wavelength $\lambda=427.8 \mathrm{~nm}$ can be obtained by pumping the pure nitrogen in the cell (at atmospheric pressure) by femtosecond laser at $\lambda=950 \mathrm{~nm}$. A little later in the same year the super-radiance at this wavelength was obtained by irradiation of pure nitrogen in the cell [14] and the air $[16,17]$ by radiation of femtosecond laser at $\lambda=800 \mathrm{~nm}$. Thus in $[11,12,15-17]$ it has been shown that as the initiation of radiation, resulting in an exponential increase of photon on laser transitions of the molecular nitrogen ions the photons of supercontinuum (laser white light) are.

International Conference on Atomic and Molecular Pulsed Lasers XII, edited by Victor F. Tarasenko,

Andrey M. Kabanov, Proc. of SPIE Vol. 9810, 98100L · C 2015 SPIE

CCC code: $0277-786 \mathrm{X} / 15 / \$ 18 \cdot$ doi: $10.1117 / 12.2228587$

Proc. of SPIE Vol. 9810 98100L-1 
Despite the fact that at present the number of experimental studies in this field is growing but the mechanisms creating an inversion population and generating in plasma of filament at the above transitions in the molecular ion of nitrogen is not completely clear. Moreover, generation and superradiance on transitions of neutral and ionized nitrogen molecular were more studied by optical pumping of air by FL operating at a wavelength of $800 \mathrm{~nm}$.

The experimental study of the mechanism of inversion population in the resonant electronic transition of $\mathrm{N}_{2}{ }^{+}$ion by the optical pumping of air and pure nitrogen by a femtosecond laser radiation at a wavelength of $950 \mathrm{~nm}$ is the aim of this work. Special attention is paid to the effective formation of the axial supercontinuum in the air.

\section{THE APPARATUS AND THE EXPERIMENT METHODS}

For the formation of the filament the radiation formed by the solid starting complex of «Start-480" created by "Avestaproject" firm was used [18]. The complex includes: Ti:Sa-driving generator with continuous pump laser, grating stretcher, regenerative and multipass amplifiers with pulsed lasers pumping at a wavelength of $532 \mathrm{~nm}$, grating compressor. On the output the laser emission has the wavelengths of $940-950 \mathrm{~nm}$, a diameter of $1 \mathrm{~cm}$, the pulse width 50 $\mathrm{fs}$, energy of $15 \mathrm{~mJ}$. The filament was formed into a focused beam in the air or in a cell with nitrogen. Focal length of the spherical mirror was changed in a wide range: from $3 \mathrm{~cm}$ to $12 \mathrm{~m}$. The spectral composition of the luminescence produced by filaments was measured in the direction of propagation of the pump radiation and across it. For measurements of spectra Ocean Optics HR 4000 spectrometer with a resolution of $0.03 \mathrm{~nm}$ in the wavelength range 250 $425 \mathrm{~nm}$ and wideband (200 - $1100 \mathrm{~nm}$ ) HR4000 spectrometer with a resolution of $0.75 \mathrm{~nm}$ were used.

\section{EXPERIMENTAL RESULTS}

We observed the filament in the air with length up to $5 \mathrm{~m}$ when a large focal length of 6-12 $\mathrm{m}$ of pump radiation was used. In this case the filament was a directed white light source (axial supercontinium (SC)) by pump radiation energy of 5-7 mJ. Figure 1 shows a photograph of a white light spot at a distance of $12 \mathrm{~m}$ from the end portion of the filament.

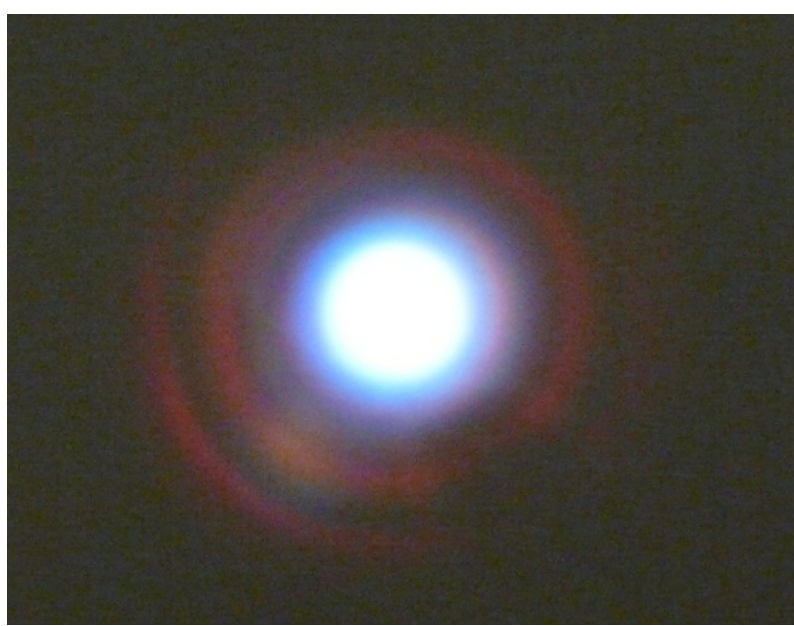

Figure 1. Photo of axial SC spots.



Figure2. The spectrum range of axial SC and the pump radiation.

According to our measurements the observed divergence of the white light is close to the diffraction limit. A typical spectrum of the radiation is shown in Figure 2. As can be seen from the figure that the spectrum has a powerful glow in the $762 \mathrm{~nm}$ comparable in intensity with the pump, and some regularly modulated structure towards the shorter 
wavelengths. With increasing of the pump energy up to $15 \mathrm{~mJ}$ the directionality of white light disappeared, the different spectral components of SC distributed at different angles relative to the axis of radiation propagation with $\lambda=950 \mathrm{~nm}$.

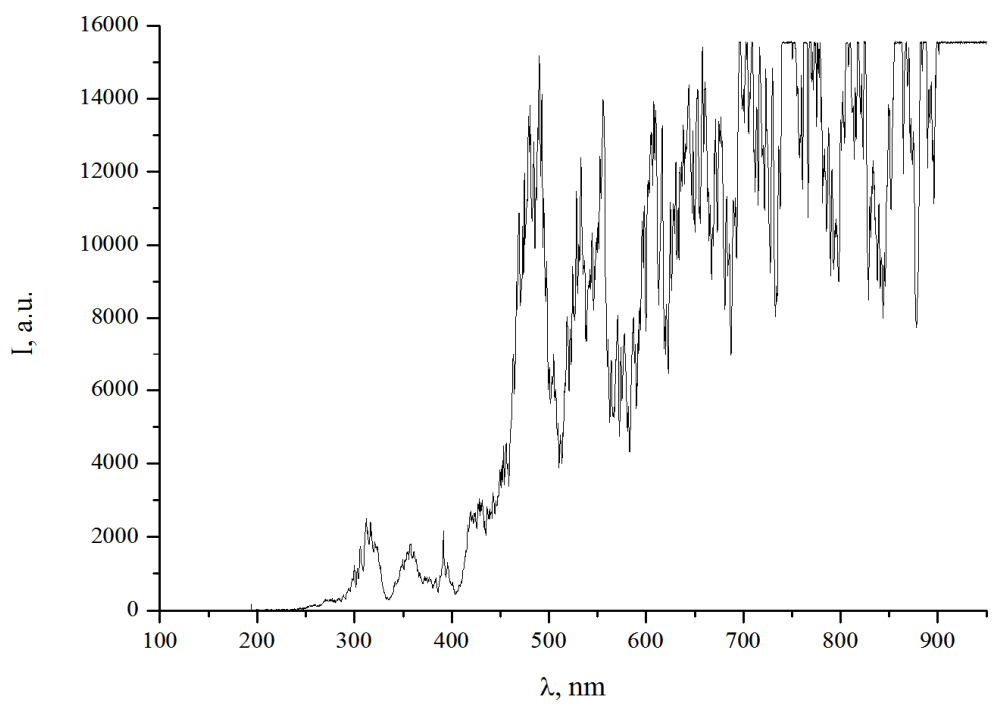

Figure 3. The spectrum of the radiation propagation in the direction of the pump. $\mathrm{F}=15 \mathrm{~cm}$.

With a decrease of the focusing length and increasing of radiation intensity in the focal range of the waist the spectrum of $\mathrm{SC}$ is broadened to the UV range up to $\lambda=300 \mathrm{~nm}$. Figure 3 shows the spectrum of the $\mathrm{SC}$ with a lens focal length $\mathrm{F}$ $=15 \mathrm{~cm}$. The spectrum modulation was irregular and it varied from pulse to pulse. In the direction of the pump radiation only conical SC is observed which had also a stochastic behavior. However, under certain conditions, first of all it is a small reduction in pump energy (12-14 $\mathrm{mJ})$ and the change in shape of the caustic of the focused radiation the directed SC ( along of axis) was registered. In this case the spectrum which was registered in the air in the direction of propagation of the pump beam strictly on axis the superradiance line of $\mathrm{N}_{2}{ }^{+}$ions with $\lambda=427.8 \mathrm{~nm}$ was observed (Figure 3,a). Under similar conditions of the pump when a filament was created in a cell with pure nitrogen gas at elevated pressure (4-6 atm) the superradiance of ions $\mathrm{N}_{2}{ }^{+} \mathrm{c} \lambda=391.4 \mathrm{~nm}$ was observed. Availability of superradiance on these lines was clearly associated with an increase in the intensity of the SC in range of $350-450 \mathrm{~nm}$, and the presence of the $3 \mathrm{rd}$ harmonic $(\lambda=317 \mathrm{~nm})$, propagating along an axis. The half-width of the superradiance lines on $\lambda=391.4$ and $\lambda=427.8 \mathrm{~nm}$ was $0.8-0.9 \mathrm{~nm}$.

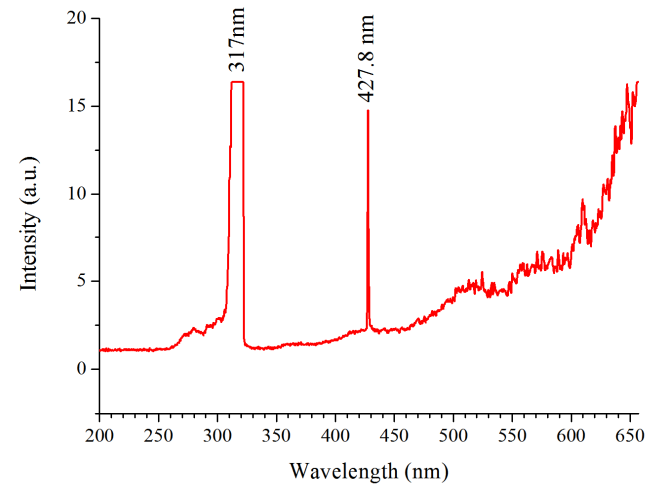

a

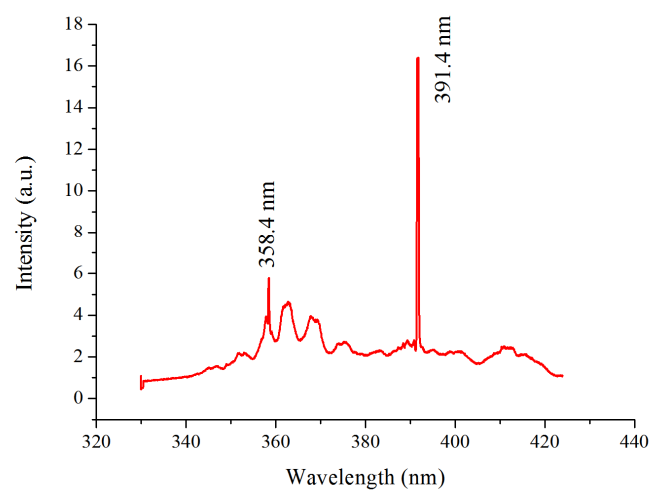

b

Figure 4. The spectra of the radiation propagating in the direction of the pump. $\mathrm{a}$ - the filaments in the air, $\mathrm{b}-\mathrm{a}$ filament in a cell with 6 bar $\mathrm{N}_{2}$. 
Simultaneously with the generation of ion transitions of molecular nitrogen the superradiance at a wavelength $\lambda=358.4$ $\mathrm{nm}$ was observed. Thus the filament was created in the cell with nitrogen under pressure of $6 \mathrm{~atm}$ and a small amount of alcohol vapor. The observed line in our opinion belongs to the transition of the $\mathrm{CN}$ molecule.

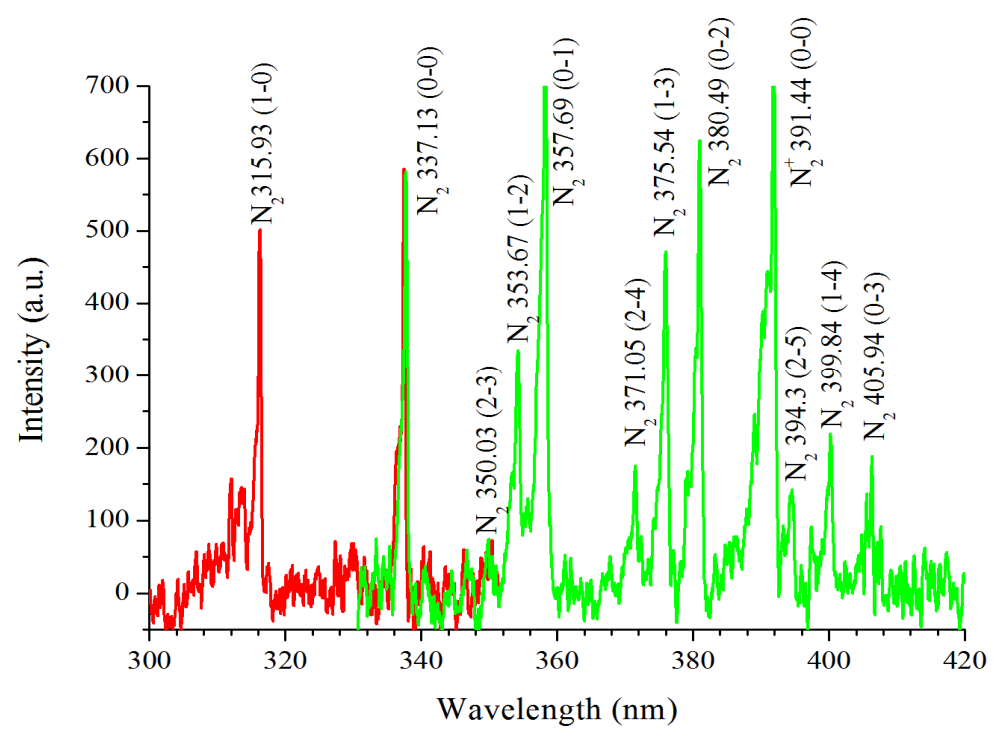

Figure 5. Emission spectrum of the filament in the perpendicular direction to its axis.

Fig. 5 shows the emission spectrum of filament in the perpendicular direction to its axis. It should be noted that in the spectrum mainly transitions to molecular nitrogen are observed. While the molecular nitrogen ion transitions are observed only in the first negative system of $\mathrm{B}^{2} \Sigma^{+}{ }_{\mathrm{u}}-\mathrm{X}^{2} \Sigma_{\mathrm{g}}^{+}$between vibration states $0-0$ and $0-1$ (the line is not represented in the figure). The intensity of the $\mathrm{N}_{2}{ }^{+}$line is comparable with the intensity of $\mathrm{N}_{2}$ lines. Both these features are not typical for the spectrum of air luminescence (nitrogen) excited by a high-voltage discharge [14].

\section{DISCUSSION}

A feature of this work is that we managed to get the super-radiance at two wavelengths of 427.8 and $391.4 \mathrm{~nm}$, using only the pump radiation with $\lambda=950 \mathrm{~nm}$. It is known that the ionization of nitrogen molecules takes place mainly through the auto-ionization (AI) states [19]. Specificity of the nitrogen molecule is that it has a state of AI which is quasi-resonant of the excited states of molecular nitrogen ion $\mathrm{B}^{2} \Sigma_{\mathrm{u}}{ }^{+}$and $\mathrm{X}^{2} \Sigma^{+} \mathrm{g}$ with vibrational number $\mathrm{v}=0$. In this case by the multiphoton ionization of nitrogen molecules by radiation of femtosecond laser with $\lambda=950 \mathrm{~nm}$ the energy of 15 pump photons exceeds the energy of the upper laser level $(18.7 \mathrm{eV}) \mathrm{B}^{2} \Sigma_{\mathrm{u}}^{+}(\mathrm{v}=0)$ at $0.88 \mathrm{eV}$ (Figure 6).

On the other hand the energy of the 12 photons practically coincides with the energy of the AI state of lower laser level $\mathrm{X}^{2} \Sigma_{\mathrm{g}}^{+}(\mathrm{v}=0)$. Since the lifetime of the AI state is tens of femtoseconds it becomes possible to obtain a population inversion for the FL radiation exposure. However to create an inversion on the transition $\mathrm{B}^{2} \Sigma_{\mathrm{u}}^{+}(\mathrm{v}=0)-\mathrm{X}^{2} \Sigma_{\mathrm{g}}^{+}(\mathrm{v}=0)$ is rather difficult. It's much easier to create an inversion on transition $\mathrm{B}^{2} \Sigma_{\mathrm{u}}^{+}(\mathrm{v}=0)-\mathrm{X}^{2} \Sigma_{\mathrm{g}}^{+}(\mathrm{v}=1)$ because the population of the first vibration level $\mathrm{X}^{2} \Sigma_{\mathrm{g}}^{+}(\mathrm{v}=1)$ is substantially smaller than the zero $\mathrm{X}^{2} \Sigma_{\mathrm{g}}^{+}(\mathrm{v}=0)$ level. In our opinion, it is linked to the observation by all authors in the air filament of the superradiance at a wavelength of $427.8 \mathrm{~nm}$, which corresponds to the transition of an electron between the vibration levels $\mathrm{v}^{\prime}=0-\mathrm{v}^{\prime \prime}=1$.

The presence of axial SC was one of the conditions of existence of superradiance in our case. Its appearance depends on the intensity of the pump. At low its intensities the axis SC exists only up to wavelengths of $450-500 \mathrm{~nm}$. At high intensities SC expands to the short side to $\lambda=300-350 \mathrm{~nm}$, but it becomes mostly conical. I. e., the conditions of the axial SC existence in the proper wavelength range is very limited. In this regard the use of ambient lighting, as was done in [12], of course is profitable. An important parameter of the superradiance on lines of 427.8 and $391.4 \mathrm{~nm}$ is its short duration which can be used in the study of fast processes. In our conditions the spectral width of these lines was similar 
and was $0.8-0.9 \mathrm{~nm}$ at half-maximum intensity. From the uncertainty relation for Gaussian beam $(\Delta \omega \times \Delta \mathrm{t} \geq 0.441)$ the pulse duration this radiation should be no less $250-350 \mathrm{fs}$. Note that in [15] this duration was $900 \mathrm{fs}$. Thus the implementation in our conditions the superradiance lines of 427.8 and $391.4 \mathrm{~nm}$ pumped by the fundamental harmonic is due, in our opinion, a number of favorable factors. Above all this is associated with a wavelength of $950 \mathrm{~nm}$. As for its photon the energy is $1.3 \mathrm{eV}$, then for multiphoton ionization of molecular nitrogen in the upper laser level $(18.7 \mathrm{eV})$ requires the 15 photons (Figure 6 ). Thus the excess energy above $\mathrm{B}^{2} \Sigma_{\mathrm{u}}^{+}(\mathrm{v}=0)$ state is $0.88 \mathrm{eV}$, while in the work with $\lambda=$ $800 \mathrm{~nm}[11,12,15-17] 13$ photons requires, which gives a total energy of $20.15 \mathrm{eV}$ and exceeding of the upper laser level at $1.45 \mathrm{eV}$. I.e. the resonance energy transfer to the upper laser level for $950 \mathrm{~nm}$ is more effective than for $800 \mathrm{~nm}$.

Besides, in our case, the multiphoton ionization can be as effectively carried out by the five photons of the third harmonic (Figure 6). We can not say it for $\lambda=800 \mathrm{~nm}$, when the third harmonic can create the upper laser level only by a five photons, but the energy excess of the upper laser level will be $4.55 \mathrm{eV}$. It is possible that more effective multiphoton ionization of nitrogen radiation with $\lambda=950 \mathrm{~nm}$ is due to our observation of superradiance at $\lambda=391,4 \mathrm{~nm}$. While in [17] it is obtained only when pumped second harmonic, or by external radiation of powerful SC. Registration of superradiance at $\lambda=391.4 \mathrm{~nm}$ by an elevated pressure of nitrogen may also due to the fact that with increasing of pressure the axial supercontium becomes conical. At the same time the long-wavelength component thereof deviates from the axis of stronger and axial intensity at $\lambda=391.4 \mathrm{~nm}$ becomes greater than $427.8 \mathrm{~nm}$.

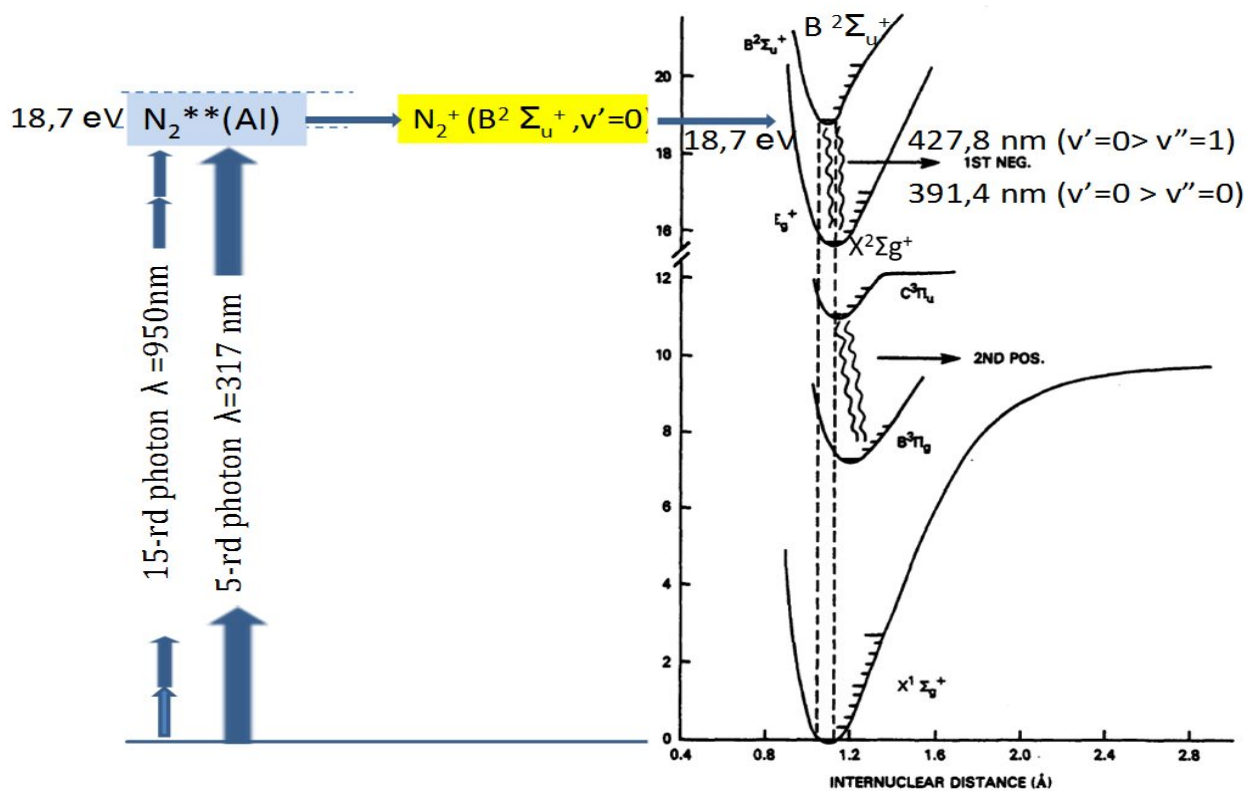

Fig.6. Potential energy curves and trajectory of the $\mathrm{N}_{2}$ and $\mathrm{N}_{2}{ }^{+}$between the energy levels.

\section{CONCLUSION}

Thus, for the first time the superradiance in the air $(\lambda=427.8 \mathrm{~nm})$ and pure nitrogen $(\lambda=391.4 \mathrm{~nm})$ on molecular nitrogen ions pumped by the femtosecond pulse of radiation at wavelengths near $950 \mathrm{~nm}$ was experimentally obtained.

It was shown that the population inversion on the $\mathrm{B}^{2} \Sigma^{+}{ }_{\mathrm{u}}-\mathrm{X}^{2} \Sigma_{\mathrm{g}}^{+}$transition of nitrogen molecular ion occurs as a result of the selective colonization of $\mathrm{N}_{2}^{+}\left(\mathrm{B}^{2} \Sigma_{\mathrm{u}}\right)$ excited state by a multiphoton excitation of autoionization states of the nitrogen molecule with energy of $18.7 \mathrm{eV}$. Seed photon for superradiance at transitions of molecular nitrogen ions are photons the axial supercontinuum occurring in the filament on the respective wavelengths.

Simultaneously with the generation on ion transitions of molecular nitrogen the superradiance regime was realized at a wavelength $\lambda=358.4 \mathrm{~nm}$. This radiation we related to transition of the $\mathrm{CN}$ molecules.

Acknowledgment: This work was supported by RFBR grants №№ 13-08-98038-r_sibir_a, 14-08-31072, 15-08-02905, 14-28-02023-ofi-m. 


\section{REFERENCES}

[1] Heard H. G., "Ultra-violet Gas Laser at Room Temperature," Nature, V.200, 667 (1963).

[2] Svedberg A., Hogborg L., Nilsson R., "Observation of superradiant laser action in spark discharges in air at atmospheric pressure," Appl. Phys. Letters. 12, 102 (1968).

[3] Antonov V. C., Knyazev I. N., Movshev V. G., "Generation UV nitrogen laser in the open air of the cuvette with transverse excitation," Quantum Electronics, V.1 (2), 433-435 (1974).

[4] Searles S. K., "Superfluorescent laser emission from electron-beam pumped $\mathrm{Ar}+\mathrm{N}_{2}$ mixtures," Appl. Phys. Lett., V.25 (12), 735-737 (1974).

[5] Collins C. B., Conningham A. J., Curry S. M., "Stimulated emission from charge-transfer reactions in the afterglow of an e-beam discharge in N2 + He high pressure mixtures," Appl. Phys. Lett., V.24 (10), 477-478 (1974).

[6] Ishchenko V. N., Lisitsyn V. N., Razhev A. M., Starinsky V. N., Chapovsky P. L., "The $\mathrm{N}_{2}^{+}$Laser," Opt. Comm., V.13 (3), 231-234 (1975).

[7] Luo Q., Liu W., Chin S. L., "Lasing action in air induced by ultra-fast laser filamentation," Appl. Phys. B., V.76, 337-340 (2003).

[8] Kartashov D., Ališauskas S., Andriukaitis G., Pugžlys A., Shneider M., Zheltikov A., Chin S. L., Baltuška A., "Free-space nitrogen gas laser driven by a femtosecond filament," Phys. Rev. A. 86 (3), 033831 (2012).

[9] Shneider M. N., Bakuška A., Zheltikov A. M., "Population inversion of molecular nitrogen in an Ar: $\mathrm{N}_{2}$ mixture by selective resonance-enhanced multiphoton ionization," J. Appl. Phys. 110 (8), 083112 (2011).

[10] Yao J., Zeng B., Xu H., Li G., Chu W., Ni J., Zhang H., Chin S. L., Cheng Y., Xu Z., "High-brightness switchable multiwavelength remote laser in air," Phys. Rev. A 84 (5), 051802 (2011).

[11] Ni J., Chu W., Jing C., Zhang H., Zeng B., Yao J., Li G., Xie H., Zhang C., Xu H., Chin S. L., Cheng Y., Xu Z., "Identification of the physical mechanism of generation of coherent $\mathrm{N}_{2}$ emissions in air by femtosecond laser excitation," Opt. Express 21 (7), 8746-8752 (2013).

[12] Yao J., Li G., Jing C., Zeng B., Chu W., Ni J., Zhang H., Xie H., Zhang C., Li H., Xu H., Chin S. L., Cheng Y., $\mathrm{Xu} \mathrm{Z}$. , "Remote creation of coherent emissions in air with two-color ultrafast laser pulses," New J. Phys. 15 (2), 023046 (2013).

[13] Prokopiev V.E., Ivanov N.G. Krivonosenko D.A., Losev V.F., "A study of elementary physical processes in the plasma regions filamentation and optical breakdown in the propagation of fs laser pulses with a wavelength of $950 \mathrm{~nm}$ in air at atmospheric pressure" Program of V Russian Conference "The interaction of highly concentrated flows of energy materials in advanced technology and medicine." - Novosibirsk, 26-29 March 2013, p. 6 (2013).

[14] Prokopev V.E., Ivanov N. G., Krivonosenko D. A., Losev V. F., "Iinvestigation of the elementary physical processes in plasma of filamentation and optical breakdown regions accompanying the propagation of the femtosecond laser pulse with wavelength of $950 \mathrm{~nm}$ in air at atmospheric pressure," Russian Physics Journal, V.56 (11), 1274-1280 (2014).

[15] Liu Y., Brelet Y., Point G., Houard A., Mysyrowicz A., "Self-seeded lasing in ionized air pumped by $800 \mathrm{~nm}$ femtosecond laser pulses," Optics Express., V. 21(19), 22791-22798 (2013).

[16] Wang T.-J., Ju J., Daigle J.-F., Yuan Sh., Li R., Leang S. Ch., "Self-seeded forward lasing action from a femtosecond Ti:sapphire laser filament in air," Laser Phys. Lett., V. 10 (125401) (2013).

[17] Wang T.-J., Daigle J.-F., Ju J., Yuan Sh., Li R., Leang S. Ch., "Forward lasing action at multiple wavelengths seeded by white light from a femtosecond laser filament in air," Physical Review A 88, 053429 (2013).

[18] Alekseev S.V., Aristov A.I., Ivanov N.G., Kovalchuk B.M., Losev V.F., Mesyats G.A., Mikheev L.D., Panchenko Yu.N., Ratakhin N.A., "Multiterawatt femtosecond laser system in the visible with photochemically driven XeF(C-A) boosting amplifier," Laser and Particle Beams, V. 31 (01), 17-21 (2013).

[19] Madden R.P., Parr A. G., "Resonance phenomena in molecular photoionization: impact of synchrotron radiation," Appl. Optics., V. 21 (2), 179-188 (1982). 